\title{
Vildagliptin prevents cognitive deficits and neuronal apoptosis in a rat model of Alzheimer's disease
}

\author{
QING-HUA MA $^{1 *}$, LIU-FANG JIANG $^{2 *}$, JIAN-LIANG MAO $^{1}$, WEN-XIN XU $^{1}$ and MIN HUANG \\ ${ }^{1}$ Department of Preventive Health, The 3rd People's Hospital of Xiangcheng District, Suzhou, Jiangsu 215134; \\ ${ }^{2}$ Department of Basic Courses, Computer Teaching and Research Section, Suzhou Vocational Health College, Suzhou, \\ Jiangsu 215009; ${ }^{3}$ Department of General Medicine, Suzhou Municipal Hospital, Suzhou, Jiangsu 215001, P.R. China
}

Received June 27, 2017; Accepted November 9, 2017

DOI: $10.3892 / \mathrm{mmr} .2017 .8289$

\begin{abstract}
Diabetes has been identified to be a risk factor for Alzheimer's disease (AD). Vildagliptin, a novel oral hypoglycemic agent, has been demonstrated to exert protective effects on the pancreas and cardiovascular system. The present study examined the potential protective effects of vildagliptin on neurons in an AD rat model. Treatment with vildagliptin improved memory deficits and decreased neuronal apoptosis in the hippocampus. The expression levels of B cell lymphoma 2 (Bcl-2) were increased, and the expression levels of caspase-3, $\mathrm{Bcl}-2$ associated $\mathrm{X}$ protein and $\mathrm{AD}$-associated proteins were decreased in the hippocampus following treatment with vildagliptin. Additionally, the AD model-induced decrease in phosphorylated $(\mathrm{p})$ protein kinase B (p-Akt), p-glycogen synthase kinase $3 \beta$ (p-GSK3 $\beta$ ), post-synaptic density 95 and synaptophysin expression was reversed. These results indicate that vildagliptin administration exerts a protective effect against cognitive deficits by reducing tau phosphorylation and increasing the expression of proteins associated with synaptic plasticity in the hippocampus. Targeting of the Akt/GSK3 $\beta$ signaling pathway may be a key mechanism in preventing the disease progression of AD.
\end{abstract}

\section{Introduction}

Alzheimer's disease (AD) is one of the most severe types of neurodegenerative disease, characterized by a decline in memory capacity and other cognitive abilities (1). The incidence of $\mathrm{AD}$ has grown rapidly in the past few decades. It was estimated in 2010 that 40 million people worldwide were

Correspondence to: Dr Min Huang, Department of General Medicine, Suzhou Municipal Hospital, 16 Baita West Road, Gusu, Suzhou, Jiangsu 215001, P.R. China

E-mail: s2huangmin@163.com

*Contributed equally

Key words: Alzheimer's disease, dipeptidyl peptidase-4 inhibitor, apoptosis, cognitive deficits living with $\mathrm{AD}$, and this figure is expected to double every 20 years (2). At present, there are no treatments that are able to completely cure AD and only symptomatic therapeutics are available $(3,4)$. The brains of patients with AD have a number of hallmark features. For instance, twisted strands of hyperphosphorylated tau would cause neurodegeneration as a consequence of structural incompleteness of microtubules, which is indeed observed in the widespread intraneuronal fibrillary tangles and intracellular formation of neurofibrillary tangles (NFTs) (5). Senile plaques consist of modified $\beta$ amyloid (A $\beta$ ) peptides which are able to induce neuronal apoptosis. $\mathrm{A} \beta$ is rapidly degraded by a number of proteases which maintain its concentration in normal neuronal cells (6). Impaired balance between $\mathrm{A} \beta$ production and clearance contribute to the amyloidogenic pathway (6). NFTs primarily consist of phosphorylated tau protein (7). Therefore, the degree of plaque deposition and synaptic plasticity in the hippocampus are critical indices for evaluating the anti-AD efficacy of potential therapeutics.

Type 2 diabetes is a risk factor for AD development and may exacerbate the progression of the disease (8). Overlapping mechanisms between type 2 diabetes and brain disorders suggest that antidiabetic drugs may have beneficial effects on brain-cell metabolism, which may be of clinical importance for the treatment of brain complications in diabetes and AD (9). Dipeptidyl peptidase-IV (DPP4) inhibitors are a class of hypoglycemic drugs used in clinical practice that have been demonstrated to improve memory in various animal models of neurodegeneration (10-12). The results of the GDMD Study in China confirmed a positive association between plasma DPP4 activity and mild cognitive impairment in elderly patients with type 2 diabetes. DPP4 inhibitors have been demonstrated to ameliorate cognitive impairment through the suppression of inflammation and oxidative stress in mouse models (13). Vildagliptin is a DPP4 inhibitor that effectively inhibits the degradation of glucagon-like peptide-1 (GLP-1). Vildagliptin was demonstrated to increase the expression and activity of GLP-1 in the peripheral blood and to reduce $\mathrm{A} \beta$, phosphorylated tau and inflammation in an $\mathrm{AD}$ mouse model (14). Thus, the use of vildagliptin to reduce plaque formation and improve synaptic plasticity warrants further investigation. In the present study, a rat model of AD was used to investigate the effects of vildagliptin on cognitive function and to examine its underlying mechanisms. In combination with 
other evidence (14), the present study indicates that vildagliptin may inhibit plaque formation and regulate synaptic plasticity in $\mathrm{AD}$, providing novel evidence to suggest the development of vildagliptin as a potential therapy for $\mathrm{AD}$.

\section{Materials and methods}

Animals. Male Sprague-Dawley rats $(\mathrm{n}=40 ; 210-230 \mathrm{~g})$ were obtained from the Animal Center of Suzhou University (Suzhou, China) and maintained in plastic cages (5 rats per cage) at $20-24^{\circ} \mathrm{C}$ and $50 \pm 10 \%$ humidity, in a 12 -h light-dark cycle with free access to food and water. All animal experiments were approved by The Institutional Review Board of Soochow University (Suzhou, China) and experimental procedures were performed in accordance with guidelines for The Care and Use of Laboratory Animals, and The National Institutes of Health (NIH) Guide for the Care and Use of Laboratory Animals (NIH Publication no. 80-23, revised 1996; NIH, Bethesda, MD, USA).

Preparation of animal models. Rats were randomly divided into the following four groups: Sham, AD, AD + low-dose vildagliptin (AD + Vilda-L) and AD + high-dose vildagliptin $(\mathrm{AD}+$ Vilda-H). Rats were anesthetized with $1 \%$ pentobarbital sodium $(40 \mathrm{mg} / \mathrm{kg})$ via intraperitoneal injection and were subsequently administered $10 \mu \mathrm{g} / \mu \mathrm{l} \mathrm{A} \beta 1-40$ via intracerebral ventricular injection. The sham group was injected with the equivalent volume of saline. All rats were subjected to the Morris water maze test to evaluate whether the AD model was successfully established from day 11-15 following the induction of $\mathrm{AD}$ and their behavioral indexes were recorded once daily. There was one mortality incidence in the $\mathrm{AD}$ group during the model establishment. Rats in the vildagliptin groups were treated with $5(\mathrm{AD}+$ Vilda-L) or $10(\mathrm{AD}+$ Vilda-H $)$ $\mathrm{mg} / \mathrm{kg}$ vildagliptin (Gulvus; Novartis International AG, Basel, Switzerland) once a day by oral gavage for 4 consecutive weeks. Following vildagliptin treatment, behavioral tests and biochemical analysis was performed.

Morris water maze (MWM) test. The spatial learning and memory of the rats was tested by MWM assessment following treatment with vildagliptin. Rats were placed in a black circular water tank (diameter, $150 \mathrm{~cm}$; depth, $60 \mathrm{~cm} ; 25^{\circ} \mathrm{C}$ ). Reference objects were placed around the pool as visual hints and were left unaltered throughout the MWM test. During the 4-day training period, rats were randomly placed into the water at any point in the four quadrants, with one test performed in every quadrant each morning for $1 \mathrm{~min}$ at every turn. The rats that did not locate the platform were placed on the platform and allowed to stand for $15 \mathrm{sec}$. Rats that successfully located the platform were additionally permitted to stand on it for $15 \mathrm{sec}$. On the 5th day, the platform was removed and the rats were allowed to swim for $1 \mathrm{~min}$. Maze performance was recorded with a video camera located above the pool, interfaced with a video tracking system (HVS Image, Buckingham, UK). The average escape latency and time spent in each quadrant in a total of five trials was recorded.

Histology. Following completion of the MWM test, rats were anesthetized with sodium pentobarbital $(60 \mathrm{mg} / \mathrm{kg})$ and euthanized by transcardial perfusion with cold PBS. The hippocampus of brain was rapidly isolated, part of the tissues was subsequently fixed with cold $4 \%$ paraformaldehyde containing $0.2 \%$ saturated picric acid for $24 \mathrm{~h}$ at $4^{\circ} \mathrm{C}$. The remaining samples were stored at $-80^{\circ} \mathrm{C}$. Paraffin-embedded sections were cut in a coronal plane at a thickness of $5 \mu \mathrm{m}$ with a microtome. Paraffin-embedded brain sections were de-paraffinized with xylene (3 times x $5 \mathrm{~min}$ ) and subsequently rehydrated prior to Nissl staining with $0.1 \%$ (w/v) cresyl violet to investigate the degree of neuronal damage in the hippocampus. The mean number of morphologically-intact neurons per $100 \mu \mathrm{m}$ length in the CA1 hippocampal area was calculated to accurately estimate the extent of neuronal damage in comparison to controls.

Western blot analysis. Frozen samples were obtained and lysed in Tissue Protein Lysis Solution (Thermo Fisher Scientific, Inc., Waltham, MA, USA) containing 5\% Proteinase Inhibitor Cocktail (Sigma-Aldrich; Merck KGaA, Darmstadt, Germany). A total of $25 \mu \mathrm{g}$ extracted protein/lane was separated on a $12 \%$ SDS-PAGE gel (Beyotime Institute of Biotechnology, Haimen, China) and transferred onto a nitrocellulose membrane (Beyotime Institute of Biotechnology) using wet transfer. The membranes were blocked in $5 \%$ non-fat milk for $2 \mathrm{~h}$ at room temperature and washed three times in PBS with Tween 20 (PBST). The membrane was subsequently incubated with the following primary antibodies overnight at $4^{\circ} \mathrm{C}$ : Rabbit polyclonal caspase-3 (1:1,000; cat. no. AB13847; Abcam, Cambridge, UK), rabbit polyclonal B cell lymphoma 2 (Bcl-2; 1:1,000; cat. no. AB59348; Abcam), rabbit monoclonal Bcl-2 associated X protein (Bax; 1:1,000; cat. no. AB32503; Abcam), rabbit polyclonal phosphorylated (p) protein kinase B (p-Akt; 1:1,000; cat. no. AB38449; Abcam), rabbit polyclonal Akt (1:1,000; cat. no. AB8805; Abcam), rabbit monoclonal glycogen synthase kinase $3 \beta$ (GSK3 $\beta ; 1: 1,000$; cat. no. AB32391; Abcam), rabbit polyclonal p-GSK3 $\beta$ (1:1,000; cat. no. AB131356; Abcam), rabbit polyclonal postsynaptic density protein 95 (PSD-95; 1:1,000; cat. no. AB18258; Abcam), rabbit monoclonal synaptophysin (1:1,000; cat. no. AB32127; Abcam), rabbit polyclonal p-tau (1:1,000; cat. no. AB109390; Abcam), rabbit monoclonal tau (1:1,000; cat. no. AB32057; Abcam), amyloid precursor protein (APP; 1:1,000; cat. no. AB32136; Abcam) and rabbit polyclonal $\beta$-actin $(1: 1,000$; cat. no. AB8227; Abcam). Following incubation with primary antibodies, the membranes were washed with PBST three times for 10 min each and subsequently incubated with polyclonal goat anti-rabbit secondary antibody (1:1,000; Abcam; cat. no. AB205718) for $2 \mathrm{~h}$ at room temperature. Protein levels were analyzed using Image J software version 1.41 (NIH) following exposure to an ECL kit (BOSTER Biological Technology, Pleasanton, CA, USA).

Statistical analysis. The data are presented as the mean \pm standard deviation. Each experiment was repeated a minimum of 3 times. The statistical analysis was performed using one-way analysis of variance, followed by the Bonferroni post hoc test, with SPSS 21.0 (IBM Corp., Armonk, NY, USA). $\mathrm{P}<0.05$ was considered to indicate a statistically significant difference. 


\section{Results}

Vildagliptin improves spatial learning and memory impairment in the AD group. The MWM test was used to assess spatial learning function. Compared with the Sham group, the escape latency in rats were administered $10 \mu \mathrm{g} / \mu \mathrm{l} \mathrm{A} \beta 1-40$ via intracerebral ventricular injection was significantly longer $(\mathrm{P}<0.01$, Fig. 1A) from day 11-15 following the induction of AD. It indicated that the AD model is modeled successfully. After four weeks the AD group still exhibited a significant spatial learning deficit compared with the sham group, and vildagliptin administration significantly reduced the escape latency compared with the AD group, particularly in the Vilda-H group (Fig. 1B). Following the 4-day training period, the platform was removed. The time spent in the target quadrant was significantly lower in the AD group compared with the sham group (Fig. 1C). The time spent in the target quadrant by the AD + Vilda-L group was significantly increased compared with the AD group. The Vilda-H group demonstrated the most significant improvement in the spatial probe test compared to the AD group $(\mathrm{P}<0.05)$.

Treatment with vildagliptin attenuates the expression of apoptosis-associated proteins and prevents neuronal cell loss. The Nissl staining method was performed to investigate neuronal alterations in the hippocampal CA1 region of rats of different groups. Hippocampal neurons in the sham group were large and conical with well-demarcated amphophilic cytoplasm and round vesicular nuclei with prominent nucleoli (Fig. 2A). Neurons in the AD group were characterized by pyknotic pyramidal cells and pronounced neuronal body shrinkage with nuclear loss (Fig. 2B). Treatment with vildagliptin reduced the AD model-induced cell loss and pyknosis, although degenerating cells with altered morphology were still observed (Fig. 2C and D). Treatment with vildagliptin exerted significant protection against AD-induced neurotoxicity, as determined by neuronal density (Fig. 2E).

Hippocampal caspase-3, Bax and Bcl-2 expression. The expression of caspase- 3 and Bax increased in the AD model compared with the sham group (Fig. $3 \mathrm{~A}$ and $\mathrm{B} ; \mathrm{P}<0.01$ ) and treatment with vildagliptin downregulated this expression. The expression level of Bcl-2 appeared to decrease in the AD model compared with the sham group (Fig. 3B). However, vildagliptin-treated rats exhibited a dose-dependent increase in hippocampal Bcl-2 expression levels by comparison with the AD group (Vilda-L and Vilda-H, $\mathrm{P}<0.05$ ).

Vildagliptin treatmentreduces the expression of $A D$-associated proteins. The expression levels of APP in the hippocampus were determined by western blot analysis. The expression of APP increased in the AD model group compared with the sham group (Fig. 4A; P<0.05) and APP expression was downregulated by treatment with vildagliptin (Vilda-L and Vilda-H, $\mathrm{P}<0.05)$. Additionally, $\mathrm{p}$-tau expression increased in the AD group compared with the sham group (Fig. 4B; $\mathrm{P}<0.05$ ). The expression levels of $\mathrm{p}$-tau were reduced following treatment with vildagliptin (Vilda-L and Vilda-H, $\mathrm{P}<0.05$ ).

Treatment with vildagliptin enhances the expression of proteins associated with synaptic plasticity. The hippocampal expression of the synapse-associated proteins synaptophysin and PSD-95 was analyzed. Levels of PSD-95 and synaptophysin in the hippocampus were significantly decreased $(\mathrm{P}<0.01)$ in the AD group compared with the sham group (Fig. 5). Treatment with vildagliptin (Vilda-H) significantly enhanced PSD-95 and synaptophysin expression levels in the hippocampus compared with the AD group $(\mathrm{P}<0.01)$. However, the low dose of vildagliptin did not significantly increase synaptophysin expression (Fig. 5B).

Vildagliptin increases Akt/GSK3 $\beta$ pathway activity. The total protein levels of Akt and GSK $3 \beta$ did not vary notably between groups. However, levels of p-Akt were significantly decreased in the hippocampus of the compared with the sham group $(\mathrm{P}<0.05)$. Rats in the AD group had increased $\mathrm{p}-\mathrm{GSK} 3 \beta$ levels compared with the rats in sham group $(\mathrm{P}<0.05)$. The administration of vildagliptin significantly increased $\mathrm{p}-\mathrm{Akt}$ (Vilda-L, $\mathrm{P}<0.05$; Vilda-H, $\mathrm{P}<0.01$ ) and reduced $\mathrm{p}-\mathrm{GSK} 3 \beta$ levels (Vilda-L, $\mathrm{P}<0.05$; Vilda-H, $\mathrm{P}<0.01$ ), in a dose-dependent manner (Fig. 6).

\section{Discussion}

$\mathrm{AD}$ is a progressive neurodegenerative disease that leads to memory impairment, aphasia, disability, visual impairment, administrative dysfunction, and personality and behavioral changes. In 1907, Alois Alzheimer's experimental observations first identified the pathological modifications of $\mathrm{A} \beta$ and tau, which are the hallmark features of AD (15). The deposition of prefibrillar and fibrillar oligomeric $\mathrm{A} \beta$, tau protein phosphorylation, synaptic loss, inflammation and glial cell activation contribute to AD pathogenesis (16). Various hypotheses have been proposed to explain the different causes of AD, but the exact mechanism remains unknown. Currently, FDA approved drugs for AD only offer symptomatic relief through the control of neurotransmitter levels and the activity of neurotransmitters, including donepezil and memantine, which affect the cholinergic and glutamatergic systems, respectively $(17,18)$. Thus, the discovery and identification of novel and effective treatments is required.

DPP4 inhibitors are a class of oral hypoglycemic agents used in monotherapy or in combination with other antidiabetic compounds. DPP4 inhibitors bind reversibly and competitively to DPP4, which indirectly enhances the levels of incretin hormones, particularly GLP-1 and gastric inhibitory polypeptide. Numerous studies have investigated the effect of DPP4 inhibitors on cognitive function. Treatment with sitagliptin was demonstrated to significantly improve the working and reference memories of diabetic rats (19); it may additionally improve the memory and hippocampal neurogenesis of high fat-fed mice (20). Furthermore, $A \beta$ deposition was delayed in an early stage AD transgenic mouse model following treatment with sitagliptin (21).

Vildagliptin has been demonstrated to significantly reduce oxidative stress in the brain, restore brain insulin sensitivity and mitochondrial function, and improve hippocampal synaptic plasticity and cognitive function in an obese rat model (22). In a streptozotocin (STZ)-induced diabetic rat model, treatment with vildagliptin significantly improved memory and learning impairments (23). Furthermore, treatment with vildagliptin 
A
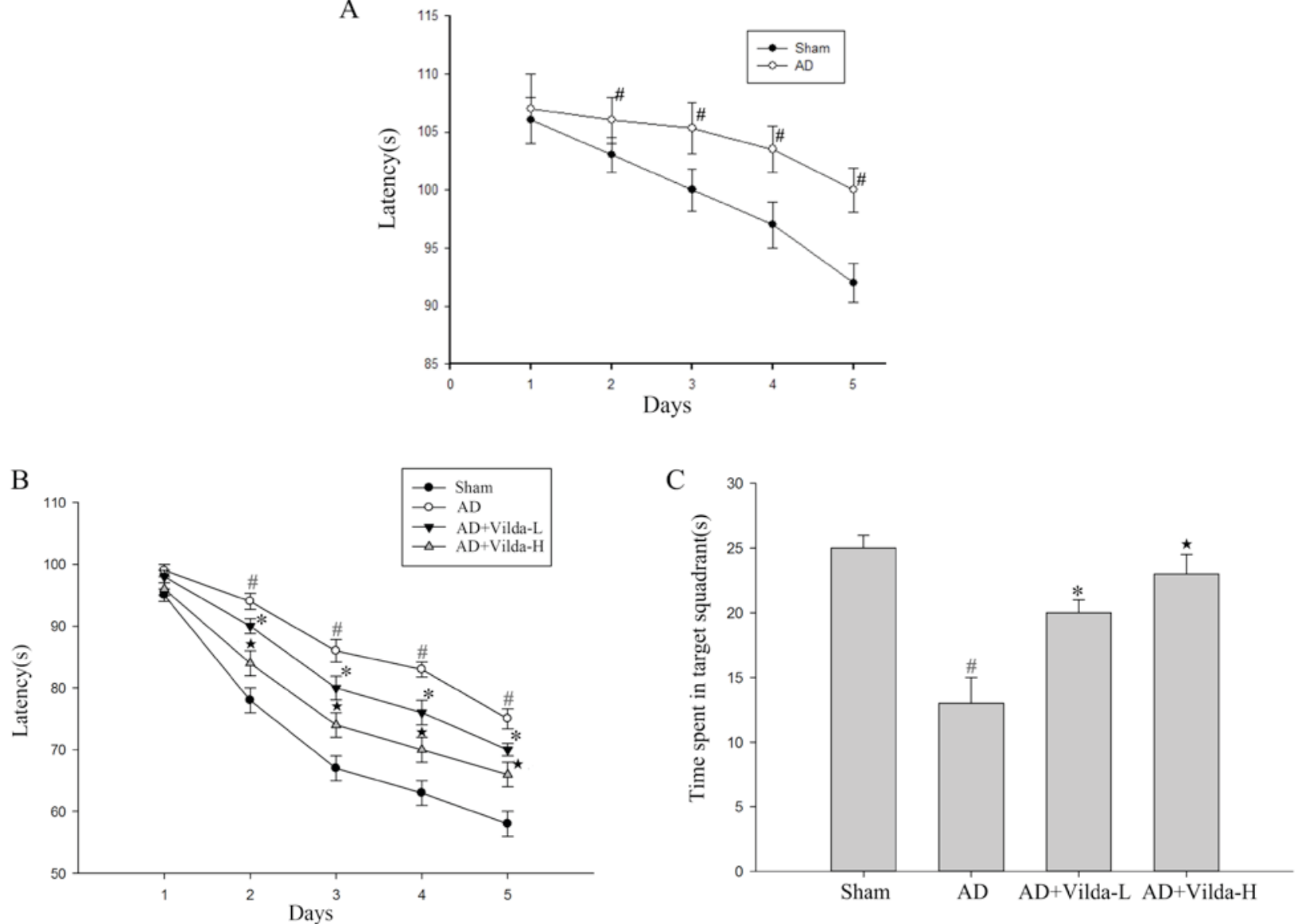

Figure 1. Results of modeling and effect of vildagliptin on AD-induced spatial learning and memory deficits measured with the Morris water maze test. (A) Results of AD model establishment. (B) Alterations in the daily escape latencies. (C) Time spent in the target quadrant when the platform was removed in the 1-min probe trial. Values are expressed as the mean \pm standard deviation ${ }^{\#} \mathrm{P}<0.01$ vs. sham; " $\mathrm{P}<0.05$ vs. AD; ${ }^{\star} \mathrm{P}<0.05$ vs. Vilda-L. AD, Alzheimer's disease model; s, sec; Vilda-L, $5 \mathrm{mg} / \mathrm{kg}$ vildagliptin; Vilda-H, $10 \mathrm{mg} / \mathrm{kg}$ vildagliptin.

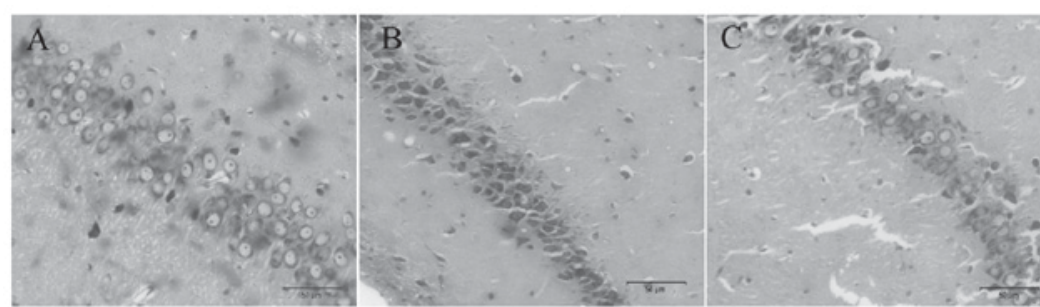

Sham
$\mathrm{AD}$

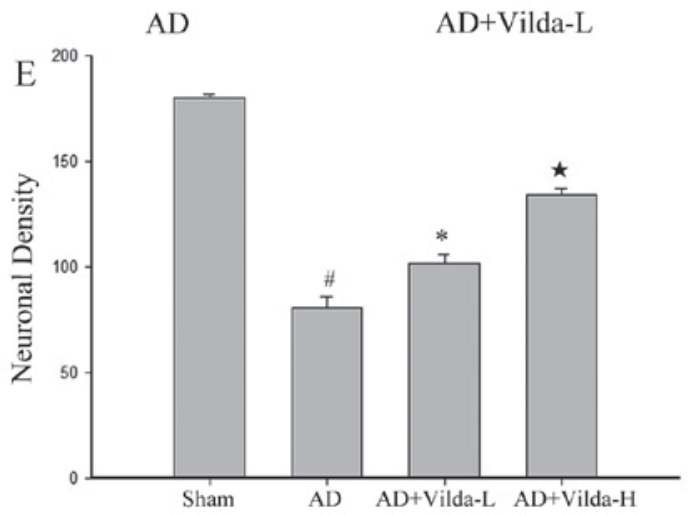

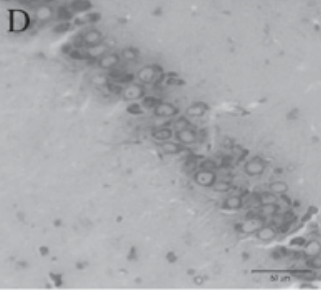

AD+Vilda-H

Figure 2. Histological analysis of the effects of vildagliptin on AD-induced neuronal injury in the hippocampal CA1 region of rats. Nissl staining was performed on sections from the hippocampal CA1 region at $x 20$ magnification. (A) Neurons with round vesicular nuclei and prominent nucleoli were observed in the sham group, with no signs of degeneration. (B) Altered neurons with pronounced neuronal body shrinkage, nuclear loss and pyknotic pyramidal cells were observed in the AD group. (C) Neuronal alteration was reduced in the Vilda-L treatment group, with few degenerating cells with altered morphology observed. (D) Vilda-H prevented neuronal cell loss. (E) The calculated neuronal density for each group. ${ }^{\prime \prime} \mathrm{P}<0.05$ vs. sham; ${ }^{*} \mathrm{P}<0.05$ vs. AD; ${ }^{\star} \mathrm{P}<0.05$ vs. Vilda-L. $\mathrm{AD}$, Alzheimer's disease model; Vilda-L, $5 \mathrm{mg} / \mathrm{kg}$ vildagliptin; Vilda- $\mathrm{H}, 10 \mathrm{mg} / \mathrm{kg}$ vildagliptin. 
A
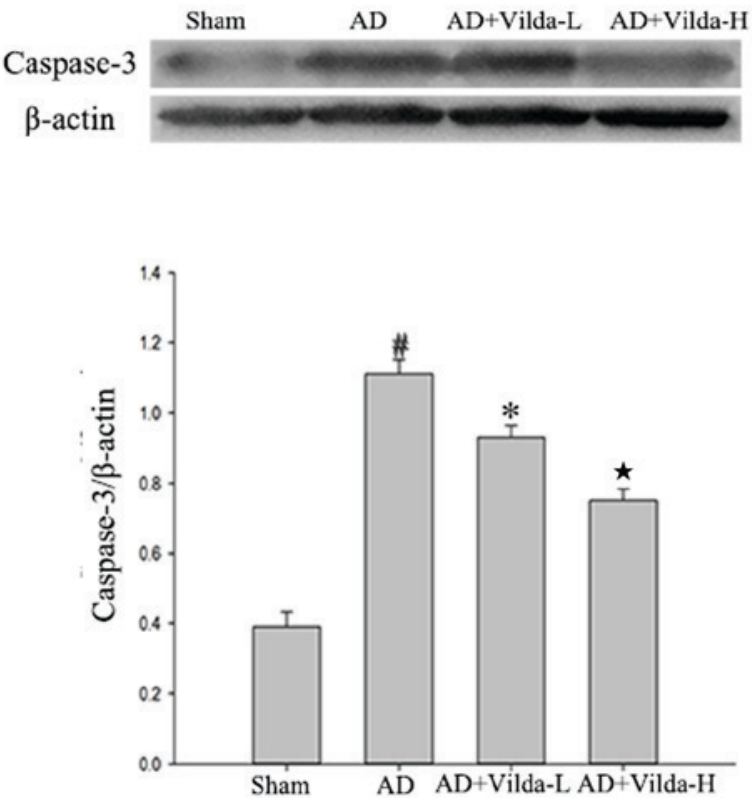

$\mathrm{B}$
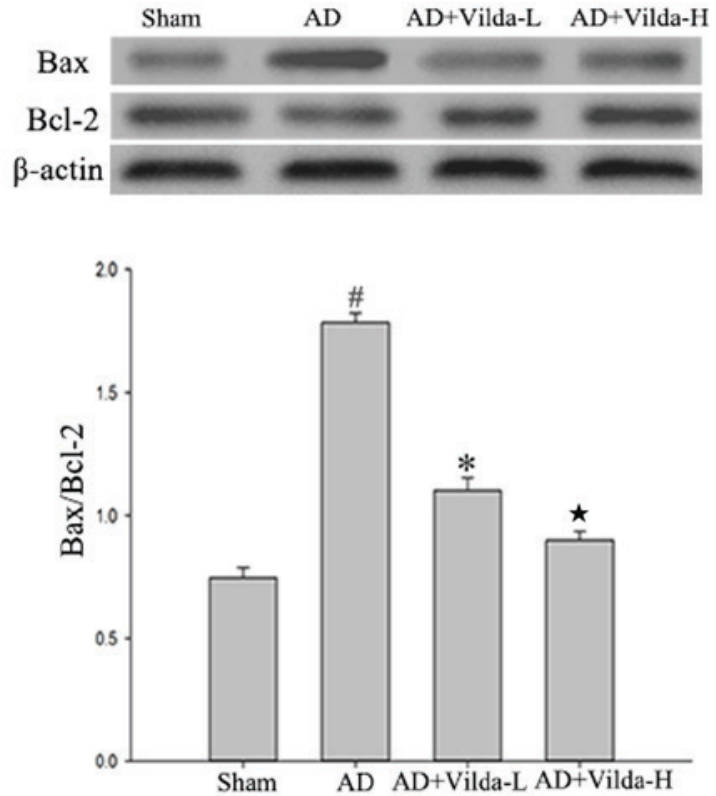

Figure 3. Effect of vildagliptin on the expression of apoptosis-associated proteins measured in rat hippocampal tissue by western blot analysis. $\beta$-actin was used as an internal control. (A) Caspase-3 expression, and (B) Bax and Bcl-2 expression. ${ }^{~} \mathrm{P}<0.01$ vs. sham group; ${ }^{*} \mathrm{P}<0.05$ vs. AD; ${ }^{\star} \mathrm{P}<0.05$ vs. Vilda-L. Bcl-2, B cell lymphoma 2; Bax, Bcl-2 associated X protein; AD, Alzheimer's disease model; Vilda-L, 5 mg/kg vildagliptin; Vilda-H, 10 mg/kg vildagliptin.

A
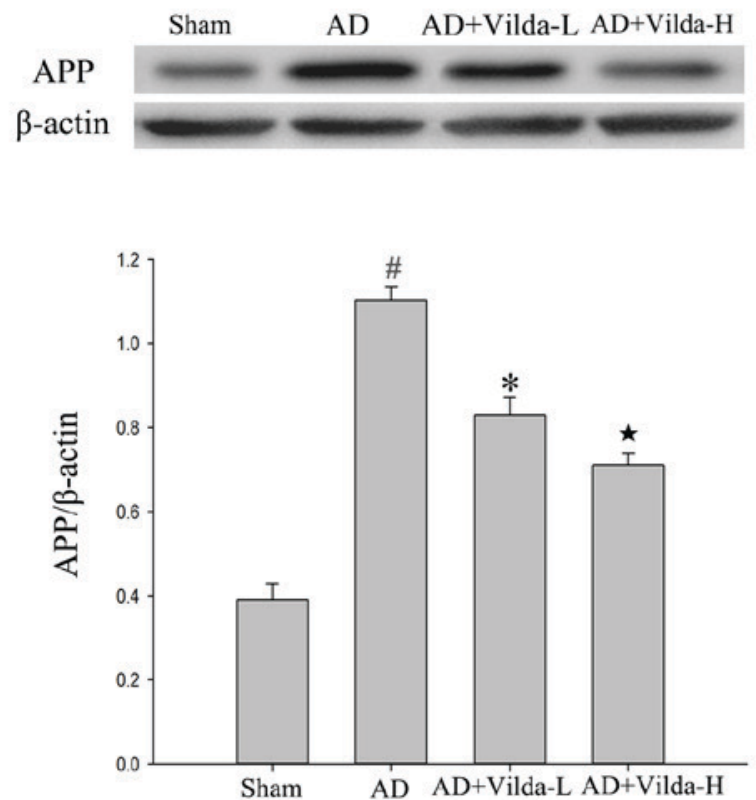

B
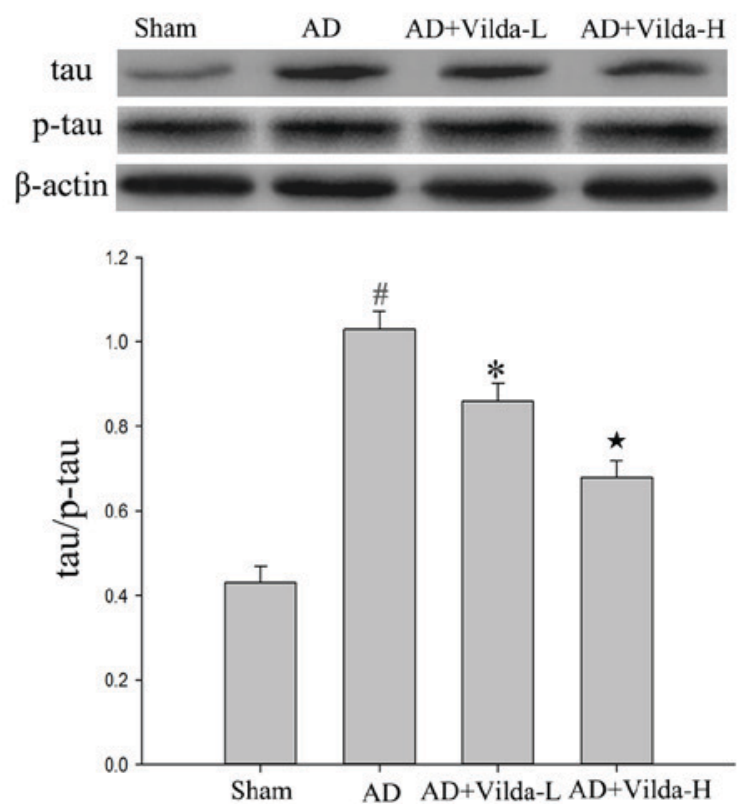

Figure 4. Effect of vildagliptin on the expression of APP and p-tau measured in rat hippocampal tissue by western blot analysis. $\beta$-actin was used as an internal control. (A) APP expression and (B) p-tau expression. ${ }^{~} \mathrm{P}<0.05$ vs. sham. ${ }^{*} \mathrm{P}<0.05$ vs. AD; ${ }^{\star} \mathrm{P}<0.05$ vs. Vilda-L. APP, amyloid precursor protein; $\mathrm{p}$-tau, phosphorylated tau; AD, Alzheimer's disease model; Vilda-L, $5 \mathrm{mg} / \mathrm{kg}$ vildagliptin; Vilda- $\mathrm{H}, 10 \mathrm{mg} / \mathrm{kg}$ vildagliptin.

ameliorated cognitive deficits in a STZ-induced rat model of AD (14). This evidence demonstrates that vildagliptin may effectively improve cognitive function.

The results of the present study demonstrated that the established AD rat model exhibited a decline in memory performance. Treatment for 1 month with vildagliptin was demonstrated to ameliorate cognitive deficits in the $\mathrm{AD}$ model in a dose-dependent manner. Bcl-2 expression was increased, and Bax and caspase-3 expression was decreased by treatment with vildagliptin. The mechanism underlying the anti-apoptotic effect of vildagliptin was not determined, but may have been mediated by the activation of the Akt/GSK3 $\beta$ signaling pathway $(24,25)$, an effect previously reported for vildagliptin in vitro (26). Consistent with this finding, vildagliptin administration following AD model-induced neuronal damage inhibited the activation of caspase-3, decreased Bax 
A
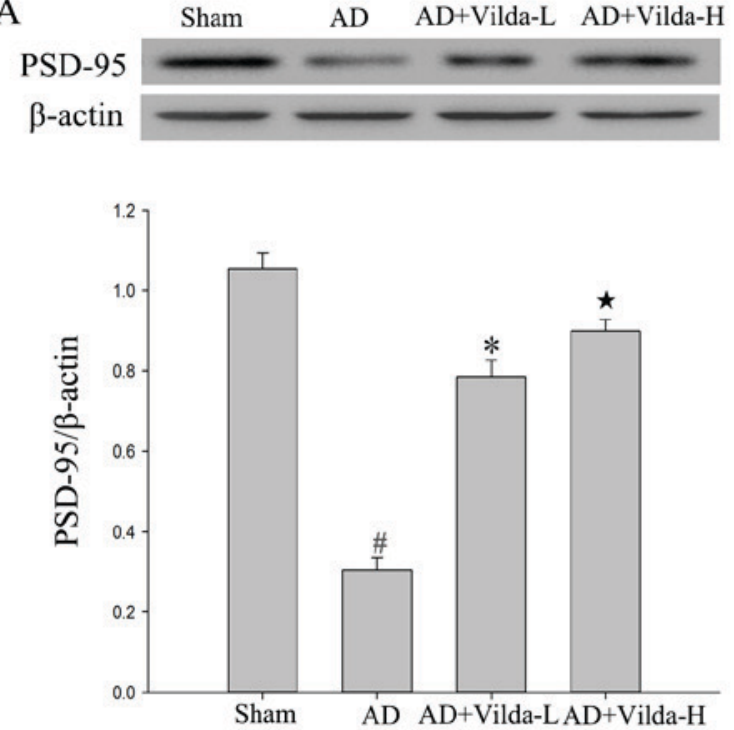

B Synaptophysin $\beta$-actin
$\mathrm{AD} \quad \mathrm{AD}+$ Vilda-L $\mathrm{AD}+$ Vilda-H

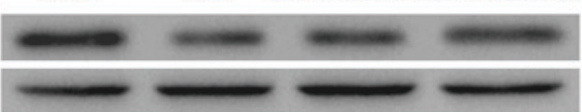

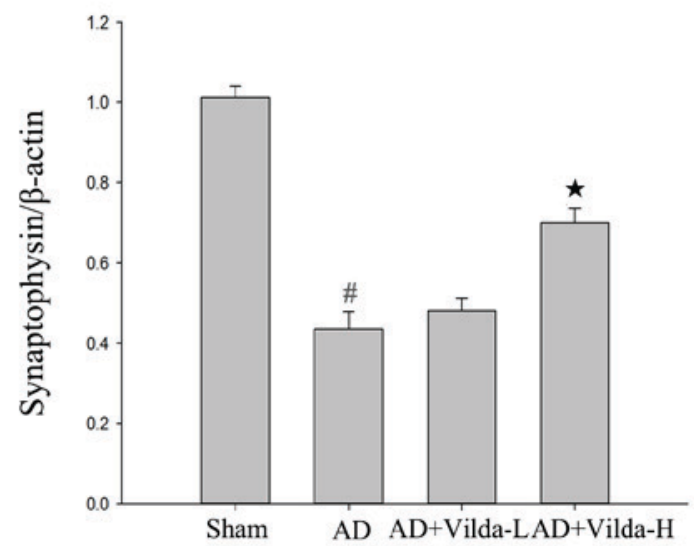

Figure 5. Effect of vildagliptin on the expression of the synaptic plasticity-associated proteins PSD-95 and synaptophysin measured in rat hippocampal tissue by western blot analysis. $\beta$-actin was used as an internal control. (A) PSD-95 expression and (B) synaptophysin expression. " $\mathrm{P}<0.01 \mathrm{vs.} \mathrm{sham;} \mathrm{"P<0.05} \mathrm{vs.} \mathrm{AD;}$ ${ }^{\star} \mathrm{P}<0.05$ vs. Vilda-L. PSD-95, post synaptic density 95; AD, Alzheimer's disease model; Vilda-L, $5 \mathrm{mg} / \mathrm{kg}$ vildagliptin; Vilda-H, $10 \mathrm{mg} / \mathrm{kg}$ vildagliptin.

A
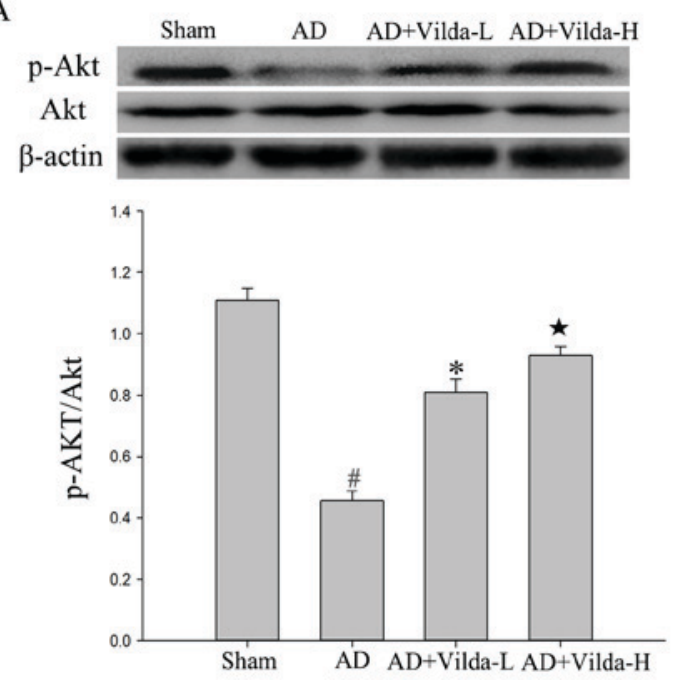

$\mathrm{B}$
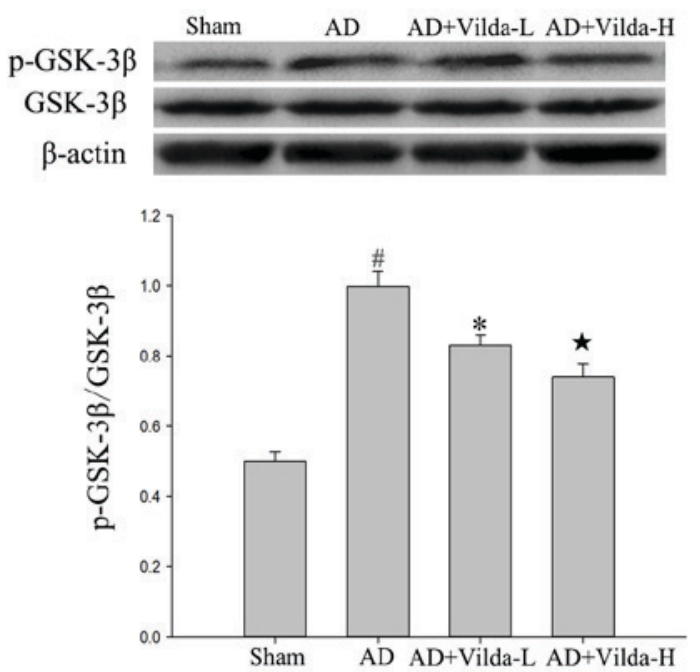

Figure 6. Effect of vildagliptin on the expression of Akt and GSK3 $\beta$ measured in rat hippocampal tissue by western blot analysis. $\beta$-actin was used as an internal control. (A) Total Akt and phosphorylated Akt expression levels, and (B) total GSK3 $\beta$ and p-GSK3 $\beta$ expression levels. " $\mathrm{P}<0.05$ vs. sham; " $\mathrm{P}<0.05$ vs. AD; ${ }^{\circ} \mathrm{P}<0.05$ vs. Vilda-L. p, phosphorylated; Akt, protein kinase B; GSK3 $\beta$, glycogen synthase kinase $3 \beta$; AD, Alzheimer's disease model; Vilda-L, $5 \mathrm{mg} / \mathrm{kg}$ vildagliptin; Vilda-H, $10 \mathrm{mg} / \mathrm{kg}$ vildagliptin.

and increased Bcl-2 expression, and therefore may reduce apoptosis in the brain.

The AD-afflicted brain is characterized by the presence of senile plaques and NFTs composed of aggregated A $\beta$ peptides and $p$-tau, respectively (27-29). It has been reported that $A \beta$ plaques are present in the brain of approximately one-third to one-half of individuals aged $\geq 65$, and tau inclusions are present almost universally (30). In AD, APP is cleaved by $\beta$-secretase and $\gamma$-secretase to produce toxic $A \beta$ protein, which is involved in plaque formation (31). In the present study, vildagliptin was demonstrated to significantly reduce APP and p-tau protein expression, with this effect being more pronounced at the higher $10 \mathrm{mg} / \mathrm{kg}$ vildagliptin dose. This suggested that vildagliptin was able to reduce $A \beta$ aggregation and tau hyperphosphorylation in
AD. Expression of PSD-95 in the AD group was significantly decreased compared with the sham group, and this expression was recovered by treatment with vildagliptin, suggesting that vildagliptin may improve synaptic plasticity, which is important for improving cognitive function. Synaptophysin expression levels were not significantly improved in the AD+Vilda-L group compared with the AD group. However, a significant improvement was observed in the $\mathrm{AD}+\mathrm{Vilda}-\mathrm{H}$ group. In terms of adverse effects, a previous study indicated that the most common adverse events in vildagliptin-treated subjects were mild or moderate, and suspected to be unrelated to the study medication. Nausea occurred in certain vildagliptin-treated subjects, although this was suggested to not be a dose-limiting side effect of DPP-4 inhibition (32). 
In conclusion, the present study demonstrated that vildagliptin improved learning and memory deficits induced in an $\mathrm{AD}$ rat model, through an increase in the expression of proteins associated with synaptic plasticity, and a decrease in the expression of apoptosis and AD-associated proteins. Additionally, activation of the Akt and GSK-3 $\beta$ inhibition may have contributed to the improvement in cognitive function mediated by vildagliptin. The present study provides evidence for the development of vildagliptin as a potential therapeutic for AD.

\section{Acknowledgements}

The present study was supported by a Project of Opening Project of Provincial Key Laboratory of Soochow University (grant no. KJS1513) and the Suzhou Science and Technology Project (grant no. XJ201460).

\section{References}

1. Scheltens P, Blennow K, Breteler MM, de Strooper B, Frisoni GB, Salloway S and Van der Flier WM: Alzheimer's disease. Lancet 388: 505-517, 2016.

2. Prince M, Bryce R, Albanese E, Wimo A, Ribeiro W and Ferri CP: The global prevalence of dementia: A systematic review and metaanalysis. Alzheimers Dement 9: 63-75, 2013.

3. Li X, Bao X and Wang R: Neurogenesis-based epigenetic therapeutics for Alzheimer's disease. Mol Med Rep 14: 1043-1053, 2016.

4. Chen PY, Tsai CT, Ou CY, Hsu WT, Jhuo MD, Wu CH, Shih TC, Cheng TH and Chung JG: Computational analysis of novel drugs designed for use as acetylcholinesterase inhibitors and histamine $\mathrm{H} 3$ receptor antagonists for Alzheimer's disease by docking, scoring and de novo evolution. Mol Med Rep 5: 1043-1048, 2012

5. Henry W, Querfurth HW and LaFerla FM: Mechanisms of disease Alzheimer's disease. New Engl J Med 362: 329-344, 2010.

6. Zuroff L, Daley D, Black KL and Koronyo-Hamaoui M: Clearance of cerebral $A \beta$ in Alzheimer's disease: Reassessing the role of microglia and monocytes. Cell Mol Life Sci 74: 2167-2201, 2017.

7. Kepp KP: Bioinorganic chemistry of Alzheimer's disease. Chem Rev 112: 5193-5239, 2012.

8. Patrone C, Eriksson O and Lindholm D: Diabetes drugs and neurological disorders: New views and therapeutic possibilities. Lancet Diabetes Endocrinol 2: 256-262, 2014.

9. Palleria C, Leporini C, Maida F, Succurro E, De Sarro G, Arturi F and Russo E: Potential effects of current drug therapies on cognitive impairment in patients with type 2 diabetes. Front Neuroendocrinol 42: 76-92, 2016

10. Darsalia V, Olverling A, Larsson M, Mansouri S, Nathanson D, Nyström T, Klein T, Sjöholm $\AA$ and Patrone C: Linagliptin enhances neural stem cell proliferation after stroke in type 2 diabetic mice. Regul Pept 190-191: 25-31, 2014.

11. Nassar NN, Al-Shorbagy MY, Arab HH and Abdallah DM: Saxagliptin: A novel antiparkinsonian approach. Neuropharmacology 89: 308-317, 2015.

12. Matteucci E and Giampietro O: Mechanisms of Neurodegeration in Type 2 diabetes and the neuroprotective potential of dipeptidyl peptidase 4 inhibitors. Curr Med Chem 22: 1573-1581, 2015.

13. Zheng T, Qin L, Chen B, Hu X, Zhang X, Liu Y, Liu H, Qin S Li G and Li Q: Association of plasma DPP4 activity with mild cognitive impairment in elderly patients with type 2 diabetes: results from the GDMD study in China. Diabetes Care 39: $1594-1601,2016$
14. Kosaraju J, Murthy V, Khatwal RB, Dubala A, Chinni S, Muthureddy Nataraj SK and Basavan D: Vildagliptin: An anti-diabetes agent ameliorates cognitive deficits and pathology observed in streptozotocin-induced Alzheimer's disease. J Pharm Pharmacol 65: 1773-1784, 2013.

15. Brion JP, Fraser H, Flament-Durand J and Dickinson AG: Amyloid scrapie plaques in mice and Alzheimer senile plaques, share common antigens with tau, a microtubule-associated protein. Neurosci Lett 78: 113-118, 1987.

16. Gonzalez B, Abud EM, Abud AM, Poon WW and Gylys KH: Tau spread, apolipoprotein E, inflammation and more: Rapidly evolving basic science in alzheimer disease. Neurol Clin 35: 175-190, 2017.

17. Storr T: Ligand design in medicinal inorganic chemistry. John Wiley \& Sons, New York, NY, 2014

18. Jakobroetne R and Jacobsen H: Alzheimer's disease: From pathology to therapeutic approaches. Angew Chem Int Ed Enge 48: 3030-3059, 2009.

19. Pintana H, Apaijai N, Chattipakorn $\mathrm{N}$ and Chattipakorn SC: DPP-4 inhibitors improve cognition and brain mitochondrial function of insulin-resistant rats. J Endocrinol 218: 1-11, 2013.

20. Gault VA, Lennox R and Flatt PR: Sitagliptin, a dipeptidyl peptidase-4 inhibitor, improves recognition memory, oxidative stress and hippocampal neurogenesis and upregulates key genes involved in cognitive decline. Diabetes Obes Metab 17: 403-413, 2015.

21. D'Amico M, Di Filippo C, Marfella R, Abbatecola AM, Ferraraccio F, Rossi F and Paolisso G: Long-term inhibition of dipeptidyl peptidase-4 in Alzheimer's prone mice. Exp Gerontol 45: 202-207, 2010

22. Pintana H, Tanajak P, Pratchayasakul W, Sa-Nguanmoo P, Chunchai T, Satjaritanun P, Leelarphat L, Chattipakorn N and Chattipakorn SC: Energy restriction combined with dipeptidyl peptidase-4 inhibitor exerts neuroprotection in obese male rats. Br J Nutr: 1-9, 2016.

23. El Batsh MM, El Batch MM, Shafik NM and Younos IH: Favorable effects of vildagliptin on metabolic and cognitive dysfunctions in streptozotocin-induced diabetic rats. Eur J Pharmacol 769: 297-305, 2015.

24. Gao C, Hölscher C, Liu Y and Li L: GSK3: A key target for the development of novel treatments for type 2 diabetes mellitus and Alzheimer disease. Rev Neurosci 23: 1-11, 2011.

25. Yu T and Lin W: Small-molecule GSK-3 inhibitor rescued apoptosis and neurodegeneration in anesthetics-injured dorsal root ganglion neurons. Biomed Pharmacother 84: 395-402, 2016.

26. Fan R, Li X, Gu X, Chan JC and Xu G: Exendin-4 protects pancreatic beta cells from human islet amyloid polypeptide-induced cell damage: Potential involvement of AKT and mitochondria biogenesis. Diabetes Obes Metab 12: 815-824, 2010.

27. Savelieff MG, Lee S, Liu Y and Lim MH: Untangling amyloid- $\beta$, tau, and metals in Alzheimer's disease. Acs Chem Biol 8: 856-865, 2013.

28. Nadezhdin KD, Bocharova OV, Bocharov EV and Arseniev AS: Structural and dynamic study of the transmembrane domain of the amyloid precursor protein. Acta Naturae 3: 69-76, 2011.

29. Köpke E, Tung Y C, Shaikh S, Alonso AC, Iqbal K and Grundke-Iqbal I: Microtubule-associated protein tau. Abnormal phosphorylation of a non-paired helical filament pool in Alzheimer disease. J Biol Chem 268: 24374-24384, 1993.

30. Braak H, Thal D R, Ghebremedhin E and Del Tredici K: Stages of the pathologic process in Alzheimer disease: Age categories from 1 to 100 years. J Neuropathol Exp Neurol 70: 960-969, 2011.

31. Do Carmo S, Crynen G, Paradis T, Reed J, Iulita MF, Ducatenzeiler A, Crawford F and Cuello AC: Hippocampal proteomic analysis reveals distinct pathway deregulation profiles at early and late stages in a rat model of alzheimer's-like amyloid pathology. Mol Neurobiol: May 13, 2017 (Epub ahead of print).

32. Pratley RE, Jauffret-Kamel S, Galbreath E and Holmes D: Twelve-week monotherapy with the DPP-4 inhibitor vildagliptin improves glycemic control in subjects with type 2 diabetes. Horm Metab Res 38: 423-428, 2006. 